\title{
PERSONAS CON DISCAPACIDAD Y SUS ROLES OCUPACIONALES: TRABAJO, FAMILIA, INDEPENDENCIA Y PARTICIPACIÓN SOCIAL
}

\author{
OCCUPATIONAL ROLES OF PEOPLE WITH DISABILITIES: WORK, FAMILY, \\ INDEPENDENCE AND SOCIAL PARTICIPATION
}

\section{Daniel Marinho Cezar da Cruz ${ }^{1}$, Vanessa Figueiredo e Silva ${ }^{2}$, Sabrina Ferigato ${ }^{3}$ y Romina Agostini ${ }^{4}$}

\begin{abstract}
Resumen
Este estudio se propone describir las necesidades cotidianas y los roles ocupacionales de un grupo de personas adultas y ancianas con discapacidad en una ciudad de medio porto del Estado de São Paulo, Brasil. Metodológicamente, se seleccionaron dieciséis usuarios con una edad media de 61,7 años y desviación estándar de 17,2 años, con discapacidad física y/o sensorial, con excepción de personas con alteraciones en el lenguaje oral y/o alteraciones cognitivas. Los instrumentos utilizados para recolectar datos fueron el Mini Examen del Estado Mental (para seleccionar la muestra sin alteraciones cognitivas), el Listado de Roles y una guía de entrevista semiestructurada. Resultados: predominan ancianos de género femenino entre las personas con discapacidad física $(n=10)$. Las entrevistas refieren que las relaciones de trabajo y funciones familiares son roles importantes, seguidos del deseo de independencia para realizar otras actividades como las de ocio, servicios domésticos y participación social. Conclusión: la investigación evidenció la importancia de mirar los roles ocupacionales en la vida cotidiana, entendiendo que la ampliación de protagonismo de las personas en su desempeño ocupacional puede favorecer su salud y el aumento de su vida social.
\end{abstract}

\section{Palabras clave:}

personas con discapacidad, roles ocupacionales, terapia ocupacional.

1 Profesor asistente Nivel 01, del Departamento de Terapia Ocupacional de la Universidade Federal de São Carlos (UFSCar). Maestría en Educación Especial por la UFSCar. Doctorado en Educación Especial y Residencia en Terapia Ocupacional en Rehabilitación Física por la Associação de Assistência à Crianza Deficiente (Asociación de Asistencia al Niño Deficiente). Dirección: Universidade Federal de São Carlos (UFSCar), Departamento de Terapia Ocupacional (DTO). Rodovia Washington Luís, s/n - Jardim Guanabara, São Carlos - SP, 13565905. Telefone: 0055-16-33518342. Email: danielcruz@ufscar.br.

2 Aluna do curso de graduação em Terapia Ocupacional pela Universidade Federal de São Carlos (UFSCar), bolsista PIBIC/CNPq/UFSCar. Membro do LAFATec, UFSCar. Dirección: Universidade Federal de São Carlos (UFSCar), Departamento de Terapia Ocupacional (DTO). Rodovia Washington Luís, s/n - Jardim Guanabara, São Carlos - SP, 13565-905. Telefone: 0055-16- 33518342. Email: vanessafes@gmail.com.

3 Professora adjunta I do Departamento de Terapia Ocupacional da UFSCar. Dirección: Universidade Federal de São Carlos (UFSCar), Departamento de Terapia Ocupacional (DTO). Rodovia Washington Luís, s/n - Jardim Guanabara, São Carlos - SP, 13565-905. Telefone: 0055-16-33518342 Email: sabrinaferigato@gmail.com.

4 Licenciada en Terapia Ocupacional. Profesora Instituto Populorum Progressio. Jujuy, Argentina. Teléfono: (54) (0342) 154238579. Email: romiagostini@hotmail.com. 


\begin{abstract}
The objective of this study is to describe the everyday needs and occupational roles of adults and old adults with disabilities in a medium port city of the Sao Paulo provincy, Brazil. Method: sixteen adults and older adults age mean 61.7 an standard deviation 17.2 participated in this study. Those selected were people with physical and/ or sensory impairment, to the exclusion of people with alterations in verbal and / or cognitive impairments. The instruments used for data collection were the Mini Mental State Examination (as a criteria to exclude subjects with cognitive imnpairments), the Role Checklist and semi-structured interviews. The results revealed the predominance of elderly females among people with disabilities $(n=10)$. The interviews showed labor relations and family functions as important roles, followed by the desire for independence in carrying out other activities such as leisure, housework and greater social participation. Conclusion: The research highlighted the importance of looking at the occupational roles of people with disabilities in their daily lives, understanding that the expansion of the role of these people in their occupational performance can promote good health and increased participation in social life.
\end{abstract}

\title{
Keywords:
}

disable persons, occupational roles, occupational therapy.

Fecha de recepción: 27/05/2016.

Fecha de aceptación: 27/10/2016

\section{INTRODUCCIÓN}

En el contexto brasilero, el análisis de Aranha (1995) refiere que la concepción de discapacidad a lo largo de la historia fue construida y reconocida por la sociedad de diferentes maneras en los distintos contextos, a partir de influencias sociales, culturales, políticas y económicas, con creencias, ideologías y políticas asistenciales también distintas (Aranha, 1995).

En Brasil, la presión social de los trabajadores, usuarios, familiares, movimientos y asociaciones en el contexto de la Reforma Sanitaria en la década del 70 tuvo como consecuencia la creación de dispositivos legales en diferentes áreas cuyo objetivo fue la inclusión social de personas con discapacidad, como así también en educación, trabajo, asistencia social y accesibilidad física (Santos, 2008).

El año 1981 es nombrado por la Organización de las Naciones Unidas (ONU) como año internacional de las personas con discapacidad. Esto repercutió en Brasil con planes que originaron cambios y un posicionamiento político diferente en lo que se refiere a garantizar derechos y ciudadanía a las personas con discapacidad; por ejemplo: Plan de Acción de la Comisión Internacional de Personas con Discapacidad (United Nations, 1980), Plan Nacional de Acción Conjunta para la Integración de la Persona Portadora de Discapacidad (Brasil, 1985; Oliveira y Amaral, 2004).

Contribuyendo a los cambios, en 2006 la ONU adoptó la Convención sobre los derechos de las personas con discapacidad, con el objetivo de promover, defender y garantizar las condiciones de vida dignas a estas personas. Brasil participó de la construcción de este documento, responsabilizándose por cincuenta ar- tículos de derechos civiles, políticos, económicos, sociales y culturales dirigidos a la población brasilera con discapacidad (Organização das Nações Unidas, 2006; Brasil, 2010; Secretaria Nacional de Promoção dos Direitos da Pessoa com Deficiência, 2012).

Cabe destacar que el artículo 6 de la Convención de las Personas con Discapacidad refuerza la importancia de este segmento de la población, reconociendo que mujeres y niñas pueden estar sujetas a formas de discriminación. Por lo tanto, propone medidas que pretenden asegurar sus derechos humanos y libertades fundamentales dispuestas en la Convención Secretaria Nacional de Promoção dos Direitos da Pessoa com Deficiência (Secretaria Nacional de Promoção dos Direitos da Pessoa com Deficiência, 2012).

De acuerdo al último censo de Brasil publicado en 2010, se identificó que del 23,9\% que poseía por lo menos una discapacidad, la discapacidad visual fue la que presentó la mayor incidencia $(18,6 \%)$, seguida por la discapacidad motora $(7 \%)$, discapacidad auditiva $(5,10 \%)$ y deficiencia mental o intelectual $(1,40 \%)$ (Secretaria Nacional de Promoção dos Direitos da Pessoa com Deficiência, 2010). La población femenina sobrepasó a la masculina en 5,3 puntos porcentuales en lo que se refiere a la relación de personas con por lo menos una de las discapacidades (visual, motora, auditiva, mental o intelectual), correspondiendo a $19.805 .367(21,2 \%)$ hombres y 25.800 .681 $(26,5 \%)$ mujeres. Estos datos pueden entenderse, en parte, por el hecho de que los hombres fallecen más temprano que las mujeres, además de la frecuencia aún más alta en relación a la presencia de discapacidades combinadas con la morbilidad en la población masculina (Ibge, 2010). 


\section{Roles ocupacionales y vida cotidiana}

Los roles ocupacionaes son definidos por la AOTA (2008) como "un conjunto de comportamientos esperados por la sociedad y moldeados por la cultura que pueden conceptualizarse y definirse posteriormente por el cliente. Los roles pueden proveer una guía para seleccionar las ocupaciones y pueden conducir a patrones de participación estereotipados y limitados" (p. 22).

La vida cotidiana presenta todos los aspectos de la individualidad y personalidad de los individuos (Heller, 1992) y se establece en una relación dinámica entre los aspectos particulares de los sujetos y aquellos que constituyen el colectivo y su contexto social (Salles y Matsukura, 2013).

El cotidiano comprende la inserción en ocupaciones de la vida diaria y se constituye por aspectos biopsicosociales, políticos, económicos y ambientales como así también por comportamientos determinados por los propios individuos en relación con la sociedad y con diferentes culturas, conformando los llamados roles ocupacionales (AOTA, 2008).

Según Cruz (2012), cuando el contexto del sujeto es atravesado por un conjunto de limitaciones propias $u$ originadas en la discapacidad, se genera un impacto en el desempeño de las ocupaciones individuales y colectivas, así como en las posibilidades de participación social y protagonismo en sus roles ocupacionales.

En la literatura, investigadores contribuyen al estudio de los roles ocupacionales de personas con discapacidad que pasan por trasformaciones significativas, debido a la complejidad de alteraciones físicas, psicológicas y sociales, entre otras. Souza, Cruz, Ferrigno, Tsukimoto y Figliolia (2013) investigaron los roles ocupacionales y el nivel de independencia de sujetos que sufrieron lesión medular y están en proceso de rehabilitación, encontrando asociación entre mayor independencia con mayor número de roles desempeñados en tiempo presente, luego de la discapacidad. Cruz, Vasconcelos, Caro, Silva y Lympius (2014) describieron los roles ocupacionales de personas con secuelas de accidente cerebro vascular $(\mathrm{ACV})$, identificando los roles ocupacionales que estos individuos desempeñaban y el grado de importancia atribuido a los mismos luego de la lesión.

Partiendo de la perspectiva de que la vida cotidiana es dinámica, singular y que conlleva necesidades específicas para el sujeto, tanto individuales como colectivas, la presente investigación puede contribuir al estudio del contexto de las personas con discapacidad ya que pretende comprender el cotidiano de estas personas a partir de sus necesidades y de la participación en roles ocupacionales como aspecto que lo estructura. Se incluye el recorte temporal como elemento importante, buscando cambios ocasionados antes, durante y después de sufrida la disfunción física (Souza, Cruz, Ferrigno, Tsukimoto y Figliolia, 2013).

\section{Objetivos}

Describir los roles ocupacionales de personas con discapacidad en el pasado, presente y futuro en relación a su grado de importancia.

Informar, a partir del relato de estas personas sobre sus roles ocupacionales, cuáles son sus necesidades cotidianas.

\section{MÉTOdO}

\section{Sujetos y métodos}

Considerando la naturaleza compleja del rol ocupacional se adoptó como forma de investigación el tipo descriptivo, con abordaje cuanti-cualitativo.

La investigación fue realizada en una ciudad del interior del Estado de São Paulo en Brasil, con una población de 240.000 habitantes aproximadamente, residiendo el 96\% en área urbana y el $4 \%$ en área rural, de acuerdo al Censo de 2010. Se seleccionaron sujetos en seis Unidades de Salud de Familia (USF). Se optó por las USF por la posibilidad de acceder a personas en su propio terreno y, así, en su vida cotidiana.

Las USF se caracterizan por ser unidades locales de atención primaria de salud que adoptan un modelo de atención y gestión denominado Estrategia de Salud de la Familia (ESF), estrategia de atención básica oficial del Sistema Único de Salud (SUS) impulsada por el Ministerio de Salud.

Entre las principales normativas de la ESF se incluyen: el trabajo en equipo de base territorial y con adscripción de los usuarios, la incorporación de agentes comunitarios de salud (ACS) en equipos interdisciplinarios, la identificación de atención básica como puerta de entrada para el SUS y su instancia privilegada para la coordinación del cuidado integral de los sujetos tanto a nivel individual como colectivo. Además, el proceso de 
trabajo tiene a la comunidad y la familia como unidades privilegiadas (Campos et al, 2008).

De estas unidades fueron elegidas dieciséis personas $(n=16)$ de acuerdo a los criterios de inclusión establecidos: adultos y ancianos con discapacidad física y/o sensorial que aceptaron participar voluntariamente de la investigación mediante la firma del consentimiento informado $(\mathrm{Cl})$. En relación a los criterios de exclusión se consideraron: personas con alteraciones en el lenguaje verbal $y / o$ alteraciones cognitivas o mentales asociadas a la discapacidad física/sensorial que impidieran la realización de las entrevistas. La muestra de esta investigación fue de conveniencia (Crosby, Salazar y Di Clemente, 2006).

\section{Instrumentos de recolección de datos}

1) Mini Examen del Estado Mental: es un test simple compuesto de preguntas que evalúan orientación, memoria inmediata, atención y cálculo, memoria de evocación y lenguaje. La evaluación, que es realizada a través de puntuaciones (puntaje máximo y puntaje paciente), ofrece material complementario y conocimiento especializado. Este instrumento fue utilizado como método de selección de la muestra, donde se evaluó si los participantes poseían alteraciones cognitivas que podrían impedir responder a las preguntas (Bertoluci, Brucki, Campacci y Juliano, 1994). Luego del análisis de los resultados de la aplicación del test cognitivo Mini Examen del Estado Mental, utilizado como criterio de inclusión de la muestra, se obtuvieron dieciséis participantes $(n=16)$ de un total de 19 sujetos.

2) Entrevista semiestructurada: se compone de preguntas predeterminadas orientadas de acuerdo al plan de investigación. Esta técnica de recolección de datos tiene como ventaja una mayor flexibilidad; el entrevistador puede repetir/esclarecer las preguntas cuando sea necesario, garantizando la comprensión. Además, ofrece la oportunidad de evaluar actitudes y registrar reacciones, y se pueden obtener datos que no se encuentran en fuentes documentadas y que también son relevantes y significativas. En esta dirección, la entrevista se vuelve una oportunidad para que el entrevistado narre los aspectos de su vida cotidiana y reflexione sobre ellos mientras los describe (Ferreira y Oliver, 2010). La guía de preguntas fue elaborada por los investigadores con el objetivo de comprender el coti- diano de los sujetos de investigación. Se hizo un recorte seleccionando las preguntas que relacionan las necesidades de la vida cotidiana con los roles ocupacionales que esta población desempeña. La guía fue contruida a partir de la literatura sobre discapacidad en Brasil y de la experiencia práctica de los investigadores en el tema. Las preguntas fueron abiertas e incluían cuestiones claves: ¿qué le gusta y/o gustaría hacer en su vida cotidiana?, coméntenos sobre su vida cotidiana, ¿qué es lo que más necesita en su vida?. La propuesta de la guía era dejar que los participantes se expresaran libremente y en la medida en que los roles aparecían en el relato, se los invitaba a comentar más sobre ellos.

3) Listado de Roles: Es un instrumento de autoevaluación creado por Oakley, Kielhofner, Barris y Reichler (1986), en base al Modelo de la Ocupación Humana. Tiene como objetivo informar los roles ocupacionales de los individuos, los que desempeñan y/o les gustaría desempeñar y la importancia de los mismos en su vida cotidiana. La lista se divide en dos partes. La primera corresponde a la evaluación de diez roles ocupacionales (estudiante, trabajador, voluntario, cuidador, amo/ama de casa, amigo, miembro de la familia, participante en organizaciones, aficionado y religioso) y un ítem "otros" para que el sujeto identifique roles que no están en la lista y que el individuo considera haber desempeñado en el pasado, que desempeña en el presente y que le gustaría desempeñar en el futuro (Oakley, Kielhofner, Barris y Reichler, 1986). En la segunda parte, los diez roles se evalúan en relación al significado que cada participante le atribuye: ninguna importancia, alguna importancia y mucha importancia. Este instrumento se validó para la población brasilera en la investigación de Cordeiro, Camelier, Oakley y Jardim (2007). Si bien el instrumento Listado de Roles requiere de un análisis y entrevista posterior a su administración para ser considerado válido, en esta investigación se adoptó la metodología de entrevista abierta con el objeto de permitir que los roles ocupacionales aparecieran libre y espontáneamente en el discurso de los participantes.

El orden de aplicación de los instrumentos fue el siguiente: Mini Mental, Listado de Roles y entrevista semiestructurada. El orden tuvo como objetivo seleccionar a los sujetos para la muestra, iniciar la identificación de roles y por último, profundizar en su análisis a partir de la entrevista. 


\section{Procedimientos para el análisis de los datos}

El análisis cualitativo se realizó en base al análisis de contenido propuesto por Bardin (2011). Este método consiste en un análisis sistemático y en etapas de los datos originales de la entrevista. Los mismos fueron codificados pasando por recortes, agregados y enumeración. El análisis de esos datos fue elaborado por tres investigadores, quienes discutieron conjuntamente las categorías temáticas. Las entrevistas fueron grabadas y transcritas para el análisis posterior (Markoni y Lakatos, 2007). Cada entrevista fue pautada por teléfono y realizada con posterioridad por una sola investigadora en el domicilio de las personas, con duración de una hora aproximadamente.

Luego de la transcripción de los datos de las grabaciones, se realizó una lectura fluctuante que permitió conocer el texto atentamente y considerar impresiones y orientaciones del investigador que lo analiza (Bardin, 2011). Luego se destacaron posibles categorías emergentes.

El análisis cuantitativo fue realizado a partir de estadística descriptiva simple, solo para los datos del Listado de Roles.

En lo que respecta a los aspectos éticos, el proyecto fue evaluado y autorizado por la Secretaría Municipal de Salud de São Carlos y aprobado por el Comité de Ética en Investigación con Seres Humanos de la Universi- dade Federal de São Carlos bajo dictamen $n^{\circ} 384.759$, Comité de Ética (CAAE) no 17931613.0.0000.5504. La recolección de datos fue realizada de acuerdo al consentimiento informado firmado por todos los participantes de la investigación, donde se permite la divulgación de los datos respetando el anonimato de los entrevistados.

\section{ReSULTADOS Y DISCUSIÓN}

\section{Características sociodemográficas de la muestra}

En la Tabla 1 se presentan los datos de las características de la muestra investigada:

Tabla 1:

Datos sociodemográficos de los participantes $(n=16)^{*}$.

\begin{tabular}{|c|c|c|c|c|}
\hline \multirow[t]{2}{*}{ Sexo } & Masculino & 5 & & Desviación estándar \\
\hline & Femenino & 11 & & \\
\hline \multirow[t]{2}{*}{ Edad } & Media & 61,7 & \pm & 17,2 \\
\hline & Mediana & 62,5 & & \\
\hline \multirow[t]{2}{*}{ Ocupación } & Activo & 2 & & \\
\hline & Inactivo & 14 & & \\
\hline \multirow[t]{2}{*}{ Escolaridad } & Media & 4,8 & \pm & 3,8 \\
\hline & Mediana & 4,0 & & \\
\hline \multirow[t]{5}{*}{ Estado civil } & Soltero & 5 & & \\
\hline & Casado & 6 & & \\
\hline & Separado & 1 & & \\
\hline & Divorciado & 0 & & \\
\hline & Viudo & 4 & & \\
\hline
\end{tabular}

*Elaboración propia. 
En la muestra presentada $(n=16)$ se observó una predominancia de la discapacidad en relación al género femenino. Ese resultado coincide con los datos del censo del año 2010 (Ibge, 2010). La población anciana fue predominante (en la media de 61,7 años). En el censo de 2010 se observó prevalencia de la población de 65 años o más, lo que sugiere que el porcentaje es alto debido al proceso de envejecimiento y los déficits funcionales relacionados (Brasil, 2010). Esos datos muestran la importancia de comprender el envejecimiento y sus consecuencias, así como identificar los roles ocupacionales a lo largo de la vida para la promoción de salud y bienestar (Rebellato, Emmel, Cordeiro y Oishi, 2015).

De acuerdo al censo de 2010, las personas con discapacidad poseen menos tasas de alfabetización ${ }^{5}$ en relación a la población total en todas las regiones brasileras, destacando las regiones Norte y Nordeste con las menores tasas (Brasil, 2010). Otra evidencia se refiere a los valores del nivel de instrucción ${ }^{6}$, donde en 2010 había un valor expresivo de la población en la categoría sin instrucción y enseñanza básica completa. Las personas con discapacidad se destacaron con $61,1 \%$.

En la Tabla 2, presentada a continuación, se observan los resultados obtenidos a partir de la aplicación del Listado de Roles. Se identifica la distribución de los roles ocupacionales a lo largo de los tres tiempos: pasado, presente y futuro. Destacamos que ninguna persona entrevistada refirió poseer otros roles.

Tabla 2:

Distribución de los roles ocupacionales a lo largo del tiempo $(n=16)^{*}$.

\begin{tabular}{|c|c|c|c|c|c|c|}
\hline \multirow{3}{*}{$\frac{\text { ROLES OCUPACIONALES }}{\text { Tipos }}$} & \multicolumn{6}{|c|}{ TIEMPOS } \\
\hline & \multicolumn{2}{|c|}{ Pasado } & \multicolumn{2}{|c|}{ Presente } & \multicolumn{2}{|c|}{ Futuro } \\
\hline & $\mathrm{N}$ & $\%$ & $\mathrm{~N}$ & $\%$ & $\mathrm{~N}$ & $\%$ \\
\hline Estudiante & 14 & $88 \%$ & 1 & $6 \%$ & 9 & $56 \%$ \\
\hline Trabajador & 15 & $94 \%$ & 2 & $13 \%$ & 14 & $88 \%$ \\
\hline Voluntario & 6 & $38 \%$ & 0 & $0 \%$ & 14 & $88 \%$ \\
\hline Cuidador & 14 & $88 \%$ & 7 & $44 \%$ & 12 & $75 \%$ \\
\hline Ama/amo de casa & 15 & $94 \%$ & 9 & $56 \%$ & 15 & $94 \%$ \\
\hline Amigo & 15 & $94 \%$ & 13 & $81 \%$ & 16 & $100 \%$ \\
\hline Miembro de familia & 13 & $81 \%$ & 16 & $100 \%$ & 16 & $100 \%$ \\
\hline Religioso & 14 & $88 \%$ & 10 & $63 \%$ & 16 & $100 \%$ \\
\hline Aficionado & 14 & $88 \%$ & 12 & $75 \%$ & 14 & $88 \%$ \\
\hline Participante en organizaciones & 2 & $13 \%$ & 0 & $0 \%$ & 8 & $50 \%$ \\
\hline Otros roles & - & - & - & - & - & - \\
\hline
\end{tabular}

*Elaboración propia.

5 "La tasa de alfabetización de una población mide el porcentaje de personas de 15 años o más de edad que sabe leer y escribir por lo menos una nota simple en su idioma" (BRASIL, 2010, p.15).

6 III“El nivel de instrucción mide la proporción de personas de 15 años o más de edad que alcanzaron determinados años de estudio" (BRASIL, 2010, p.17).
Los roles más desempeñados en tiempo presente son: amigo, miembro de familia, religioso y aficionado.

Otro dato relevante es que se observa la asignación de gran valor a la mayoría de los roles ocupacionales. Esto se puede relacionar con los resultados de la Tabla 3 que presenta los valores correspondientes al grado de importancia para cada rol ocupacional: 
Tabla 3:

Distribución del grado de importancia de los roles ocupacionales $(n=16)^{*}$.

\begin{tabular}{|c|c|c|c|c|c|c|}
\hline \multirow{3}{*}{$\begin{array}{c}\text { ROLES OCUPACIONALES } \\
\text { Tipos }\end{array}$} & \multicolumn{6}{|c|}{ GRADO DE IMPORTANCIA } \\
\hline & \multicolumn{2}{|c|}{ Ninguna } & \multicolumn{2}{|c|}{ Alguna } & \multicolumn{2}{|c|}{ Mucha } \\
\hline & $\mathrm{N}$ & $\%$ & $\mathrm{~N}$ & $\%$ & $\mathrm{~N}$ & $\%$ \\
\hline Estudiante & 1 & $6 \%$ & 2 & $13 \%$ & 13 & $81 \%$ \\
\hline Trabajador & 0 & $0 \%$ & 2 & $13 \%$ & 14 & $88 \%$ \\
\hline Voluntario & 1 & $6 \%$ & 4 & $25 \%$ & 11 & $69 \%$ \\
\hline Cuidador & 0 & $0 \%$ & 3 & $19 \%$ & 13 & $81 \%$ \\
\hline Amo/ama de casa & 0 & $0 \%$ & 3 & $19 \%$ & 13 & $81 \%$ \\
\hline Amigo & 0 & $0 \%$ & 1 & $6 \%$ & 15 & $94 \%$ \\
\hline Miembro de familia & 0 & $0 \%$ & 1 & $6 \%$ & 15 & $94 \%$ \\
\hline Religioso & 0 & $0 \%$ & 2 & $0 \%$ & 14 & $88 \%$ \\
\hline Aficionado & 1 & $6 \%$ & 2 & $13 \%$ & 13 & $81 \%$ \\
\hline Participante en organizaciones & 4 & $25 \%$ & 1 & $6 \%$ & 11 & $69 \%$ \\
\hline Otros roles & - & - & - & - & - & - \\
\hline
\end{tabular}

*Elaboración propia

En la investigación de Navarro, Stoffel y Nickel (2013) con personas con secuelas de enfermedades neurológicas, también se observó la existencia de alto grado de importancia a los roles ocupacionales a pesar de que los participantes no estuvieran desempeñándolos en el presente.

\section{Roles ocupacionales: datos cualitativos}

\section{CATEGORÍA 1.- Necesidades cotidianas: el rol de trabajador, miembro de familia y cuidador}

"Quería continuar vendiendo servilletas para ayudarme" (P2).

"Me gustaría trabajar para ayudar a mi madre. ¡Necesito que algo salga bien en mi vida! Conseguir una oportunidad, ¿no? Oportunidad de empleo, ¿no?!" (P13).

"Me gustaría volver a mi trabajo, trabajar de chofer. Preciso salud y trabajo y... (pausa) pasear, ah, e ir a la cuidad, encontrar a mis amigos, tener diversión" (P15).

"Trabajar en casa, cuidar la casa" (P16).
Se percibe que las necesidades de los sujetos entrevistados se expresan en cuestiones relativas a la salud, como era esperado, pero no se limitan a ese aspecto. Las necesidades, según Souza y Pimentel (2012), "son individualmente sentidas y biológica y socialmente determinadas; su atención, satisfecha socialmente, es señal de su reconocimiento" (p. 231). Desde esta perspectiva, la salud es influenciada por los contextos socioculturales (historia, valores, deseos), así como por las condiciones de vida que no se limitan a la prevención y asistencia (Othero y Ayres, 2012).

En el análisis de los relatos se observaron los diferentes significados dados al trabajo, por ejemplo, subsistencia, interacción social y rol social. En vistas a que el trabajo fue un rol destacado en los relatos, se puede hacer un análisis reflexivo sobre las políticas referidas a la inserción de estas personas en el mercado laboral, pensando en alternativas más allá de las existentes para promover esta participación.

Hay que añadir las condiciones para volver al trabajo por parte de adultos y ancianos, quienes suelen estar capacitados pero restringidos a la jubilación por la incesante necesidad del mercado de nuevas habilidades 
y la restricción de edad consideradas productiva por las leyes y políticas existentes.

El Artículo 27 dispuesto en la Convención sobre los Derechos de las Personas con Discapacidad refuerza el Artículo 23 de la Declaración Universal de los Derechos Humanos reafirmando el derecho al trabajo de toda persona, en condiciones satisfactorias, así como la protección contra el desempleo. Además, asegura las condiciones necesarias de accesibilidad para las personas con discapacidad en Brasil (Secretaria Nacional de Promoção dos Direitos da Pessoa com Deficiência, 2012).

En esta perspectiva, pensando en aquellos que se encuentran aún en edad productiva, existe en Brasil la Ley de Cuotas creada en 1991 que establece que la empresa con 100 o más empleados tiene la obligación de emplear de 2 a 5\% de los cargos con personas con discapacidad (Brasil, 1991). Asimismo, en 2010 de acuerdo al censo había 44.073.377 personas en edad activa con por lo menos una discapacidad, pero 23,7 millones no estaban ocupadas. La existencia de leyes, sin embargo, no garantiza por completo el acceso y la permanencia de las personas con discapacidad en el trabajo, y tampoco garantiza ambientes de trabajo inclusivos (Tanaka y Manzini, 2005).

Tanaka y Manzini (2005) refieren la falta de escolaridad como una de las dificultades para el ingreso de personas con discapacidad en el mercado de trabajo. Ese dato no es sorprendente, siendo el problema de la escolarización en el país una preocupación para todos los ciudadanos, tengan o no discapacidad.

Otro aspecto interesante, en este sentido, está en el trabajo con el objetivo de ayudar a la madre, presente en el discurso de P13. Se observa la transición de roles ocupacionales o la asociación de un rol con otro; en este caso, el rol de trabajador con el de miembro de familia.

"Me gustaría tener salud para ir a Pernambuco a visitar a mi familia, me gustaría también ir a Sao Paulo a visitar también a la familia que tengo allá" (P5).

"Me gustaría viajar al Norte para visitar a la familia que hace tiempo no veo, tengo mucha nostalgia" (P7).

"Me gustaría ayudar a los nietos, mirarlos, cuidarlos" (P1).
Los roles ocupacionales se modifican a lo largo de la vida; en el proceso de desarrollo puede darse un aumento del número de roles y un descenso con el final de la vida. Sin embargo, los ancianos también pueden asumir otros roles significativos como estudiar en la universidad o desarrollar trabajos voluntarios, entre otros (Cruz y Emmel, 2012, Cruz y Emmel, 2013).

Se constató que el rol miembro de familia tiende a ser desempeñado en los tres tiempos y se le atribuye un grado de mucha importancia para la vida cotidiana de los sujetos en otras investigaciones. En el estudio de Cruz y Emmel (2012) con adultos y ancianos con discapacidad física, el rol miembro de familia estuvo entre los roles con mayor porcentaje en tiempo presente y también fue el de mayor "grado de importancia" para ambos grupos (adultos y ancianos). Santi, Mariotti y Cordeiro (2012) destacan la relevancia del rol miembro de familia, cuyos valores obtenidos en tiempo presente se mantienen estables en tiempo futuro. Algo similar ocurre en la investigación de Parreira, Cavalcanti, Cunha, Cordeiro (2013) con individuos con patologías reumatológicas, donde el rol miembro de familia tiene importancia en el presente y pretensión de desempeño en el futuro, ya que se presume que este rol ejerce influencia en el enfrentamiento de la enfermedad como apoyo social fundamental.

\section{CATEGORÍA 2.- Necesidades cotidianas: roles de independencia y participación social}

"Andar, caminar como lo hacía, ir a la panadería a comprar pan, hacer compras, ir al centro de la cuidad a comprar cosas, eso me gustaría hacer, hoy no lo hago porque no estoy en condiciones $(\ldots)^{\prime \prime}(P 8)$.

"Necesitaria ser un poco más independiente, como... para conseguir hacer mi terapia fisica, hacer mis ejercicios, y para tener un poco mas de libertad en términos de salir, ir y venir"(P9). 
"Me gustaría salir más de casa, tener más contacto con las personas; últimamente ando solo" (P14).

"Me gustaría tomar clases de gimnasia, aunque sea sólo para conversar, que pueda hacer lo mismo que ya hice no? Me gustaría también tener alguna cosa para ocupar mi tiempo, sin ser el trabajo del día a día" (P4).

"Me gustaba andar dentro de mi casa, yo necesito salud" (P12).

Los relatos anteriores sustentan el hecho de que las personas con discapacidad física pueden vivenciar, además de las consecuencias por las pérdidas de orden motor, situaciones de aislamiento y empobrecimiento de la red social (Ferreira y Oliver, 2010).

Las personas con discapacidades adquiridas pueden experimentar cambios aún mayores en la convivencia social, reduciendo su participación asociada a la cantidad y calidad de las relaciones (Ferreira y Oliver, 2010). Sustenta esta hipótesis, el estudio de Aoki, Oliver y Nicolau (2011) que tuvo como objetivo la realización del diagnóstico situacional de la condición de vida de personas con discapacidad en Brasil, donde el aislamiento domiciliar fue un dato importante.

Otro aspecto destacado se encuentra en el relato de P4, donde se percibió la importancia de las ocupaciones como promotoras de interacción social y relacionadas al uso del tiempo. A partir de la comprensión del tiempo como recurso fundamental para los sujetos, Emmel, Paganelli y Valio (2015) estudiaron el uso del tiempo de 51 ancianos saludables con el objetivo de identificar las actividades de esta población y toda la complejidad que ello engloba. Los resultados demostraron que $43 \%$ de los ancianos declararon estar satisfechos con la forma como ocupaban su tiempo, especialmente en relación a las actividades de placer.

Los mismos autores consideran que las ocupaciones están relacionadas a la construcción de la identidad personal. De esta forma, las personas escogen las ocupaciones que le son más significativas (Emmel, PaganeIli y Valio, 2015). Especialmente en esta fase de la vida, el envejecimiento puede suponer el uso de tiempo más libre y agradable. Sin embargo, en la investigación, a esta fase del desarrollo se suma la discapacidad por lo que la discusión comprende otras dimensiones.
En este contexto, los relatos de P8 y P12 refieren a la participación y desempeño en ocupaciones relacionadas a la condición de salud, o sea, los dos sujetos tienen la percepción de que la presencia de la discapacidad es determinante para la no participación en ciertas ocupaciones.

De hecho, involucrarse en ocupaciones variadas estructura la vida cotidiana y contribuye a la salud (AOTA, 2008), pero, en este caso, se puede inferir que los discursos refieren la necesidad de adaptación a la nueva condición de salud impuesta por la discapacidad.

En contrapartida, P9 expresa la condición de salud en cuanto a una posibilidad de mayor independencia, relatando el desempeño de actividades relacionadas a los cuidados. A partir de esto, se infiere que la forma en que el individuo interpreta su condición de salud se refleja directamente en sus elecciones e implicación en las ocupaciones.

De modo general, fue observado que las necesidades de los participantes y las actividades significativas que desean, influyen en los roles ocupacionales que desempeñan. Por lo tanto, la privación de estos roles se reflejó significativamente en el empobrecimiento de la vida cotidiana de los sujetos investigados y en el relato de la dificultad para satisfacer parte de sus necesidades personales.

\section{CONCLUSIONES}

La investigación evidenció la importancia de la mirada sobre los roles ocupacionales de personas con discapacidad, entendiendo que el desempeño puede favorecer la mejora de su condición de salud, la construcción de ciudadanía y el aumento de la participación en la vida social.

Desempeñar roles también significó para estas personas ampliar sus relaciones sociales y sentirse más independientes en las actividades que desean y les gustaría realizar.

Cabe aclarar que nuestra investigación presenta limitaciones: los resultados no pueden ser generalizados para la población total de personas con discapacidad, por tratarse de una muestra reducida. Sin embargo, a partir de este recorte, se pudo comprender el impacto de la discapacidad en el desempeño de roles ocupacionales de esta población lo que puede contribuir al enriquecimiento de la discusión del tema en diferentes contextos. 
Otra limitación radica en que en Brasil, de los instrumentos existentes sobre el Modelo de la Ocupación Humana, sólo el Listado de Roles fue validado para el uso en nuestro país. Esto dificulta la utilización de otros instrumentos relacionados a éste.

\section{REFERENCIAS BIBLIOGRÁFICAS}

Aoki M., Oliver F.C., Nicolau S.M. (2011). Considerações acerca das condições de vida das pessoas com deficiência a partir de um levantamento em uma unidade básica de saúde de um bairro periférico do município de São Paulo. Revista O Mundo da Saúde, 35, (2), pp. $169-178$.

AOTA. American Occupational Therapy Association (2008). Ocuppational Therapy practice framework: domain \& process. The American Journal of Occupational Therapy, 62 (6), pp. 625-683. DOI: 10.5014/ajot.62.6.625

Aranha M.S.F. (1995). Integração social do deficiente: análise conceitual e metodológica. Temas psicología. Recuperado en: http://pepsic. bvsalud.org/pdf/tp/v3n2/v3n2a08.pdf

Bardin L. (2011). Análise de conteúdo. São Paulo: Edições 70.

Bertoluci P.H.F., Brucki S.M.D., Campacci S.R., Juliano Y. (1994). O mini-exame do estado mental em uma população geral: impacto da escolaridade. Arq. Neuropsiquiatra, 52 (1), pp 1-7. http://dx.doi. org/10.1590/S0004-282X1994000100001

Brasil (1991). Lei de cotas $n^{\circ}$. 8213, de 24 de Julho de 1991. Dispõe sobre os Planos de Benefícios da Previdência Social e dá outras providências. Recuperado En: http://www.planalto.gov.br/ccivil_03/ Leis/L8213cons.htm

Brasil (2010). Ministério da Saúde. Política Nacional de Saúde da Pessoa com Deficiência. 1 ed. Brasília: Editora do Ministério da Saúde. Recuperado em: http://bvsms.saude.gov.br/bvs/publicacoes/politica_nacional_pessoa_com_deficiencia.pdf

Brasil (1985). Câmara dos Deputados, decreto de lei 91872, de 04 de novembro de 1985. Diário Oficial da União - Seção 1 - 5/11/1985, Página 16114 (Publicação Original).

Campos G.W.S., Gutierrez A.C., Guerrero A.V., Cunha G.T. Reflexões sobre a Atenção Básica e a Estratégia de Saúde da Família. In: Campos G.W.S., Guerrero A.V (Orgs). Manual de Práticas de Atenção Básica - Saúde Ampliada e Compartilhada. São Paulo: Aderaldo \& Rothschild, 2008.

Cordeiro J.R, Camelier A., Oakley F., Jardim J.R. (2007). Cross-cultural reproducibility of the Brazilian Portuguese Version of the Role Checklist for Persons With Chronic Obstructive Pulmonary Disease. American Journal of Occupational Therapy, 61, 33-40. 10.5014/ajot.61.1.33
Crosby R.A., Salazar L.F., DiClemente R.J. (2006). Principles of sampling. In: Crosby R.A., Salazar L.F., DiClemente R.J. (Editors). Research methods in health promotion.Jossey-Bass: San Francisco, pp.289-316.

Cruz D.M.C. (2012) Papeis ocupacionais e pessoas com deficiências físicas: independência, tecnologia assistiva e poder aquisitivo. São Carlos: Universidade Federal de São Carlos. Programa de Pós-Graduação em Educação Especial, Tese de Doutorado.

Cruz D.M.C., Emmel M.L.G. (2013). Associations among occupational roles, independence, assistive technology, and purchasing power of individuals with physical disabilities. Revista Latino-Americana de Enfermagem, 21(2), pp.484-491. https://dx.doi.org/10.1590/ S0104-11692013000200003

Cruz D.M.C., Emmel M.L.G. (2012). Papéis ocupacionais de pessoas com deficiências físicas: diferenças de gênero e ciclos de desenvolvimento. Revista Baiana de Terapia Ocupacional, 1 (1), pp 4-24.

Cruz D.M.C., Vasconcelos F.E.O., Caro C.C., Silva N.S., Lympius J. (2014). Entre perdas e ganhos: os papéis ocupacionais pós-acidente vascular encefálico. Revista FSA, 11 (2), pp 329-349. http:// dx.doi.org/10.12819/2014.11.2.18

Emmel M.L.G., Paganelli L.O., Valio G.T. (2015). Uso do tempo de um grupo de idosos do município de São Carlos (SP), Brasil. Revista Kairós Gerontologia, 18(2), pp. 421-442. Recuperado en: http://revistas.pucsp.br/index.php/kairos/article/view/28388/19956

Ferreira T.G., Oliver F.C. (2010). A atenção domiciliar como estratégia para ampliação das relações de convivência de pessoas com deficiências físicas. Revista Terapia Ocupacional Universidade São Paulo, 21(3), pp 189-197. http://dx.doi.org/10.11606/issn.22386149.v21i3p189-197

Heller A (1992). Estrutura da vida cotidiana. O cotidiano e a história. São Paulo: Paz e Terra, pp. 17 - 41.

Ibge (2010). Instituto Brasileiro de Geografia e Estatística. Sinopse do Censo de 2010. Recuperado en: http://www.censo2010.ibge.gov. br/sinopse/

Marconi M.A., Lakatos E.M. (2007). Técnicas de pesquisa: planejamento e execução de pesquisas, amostragens e técnicas de pesquisas, elaboração, análise e interpretação de dados. São Paulo, Atlas.

Navarro E.J., Stoffel D.P., Nickel R. (2013). A independência funcional e a manutenção dos papéis ocupacionais em sujeitos com sequelas neurológicas. Cogitare Enfermagem, 18 (4), pp 6. http://dx.doi. org/10.5380/ce.v18i4.34919

Oakley F., Kielhofner G., Barris R., Reichler R.K. (1986).The role checklist: development and empirical assessment of reliability. The Occupational Therapy Journal Research, 6 (3), pp 157-170.

Oliveira M.A.M., Amaral C.T. (2004). Políticas Públicas Contemporâneas para Educação Especial: inclusão ou exclusão?. Recuperado en: http://www.clickciencia.ufscar.br/portal/edicao21/Artigo.pdf>.

Organização das Nações Unidas. Convenção sobre os direitos das pessoas com deficiência. 2006. Recuperado en: http://www.un.org/ disabilities/convention/conventionfull.shtml 
Othero M.B., Ayres J.R.C.M. (2012). Necessidades de saúde da pessoa com deficiência: a perspectiva dos sujeitos por meio de histórias de vida. Interface - Comunicação, Saúde, Educação, 16 (40), pp 15. http://dx.doi.org/10.1590/S1414-32832012005000010

Parreira M.M., Cavalcanti A., Cunha J.H.S., Cordeiro J.J.R. (2013). Papéis ocupacionais de indivíduos em condições reumatológicas. Revista de Terapia Ocupacional Universidade de São Paulo, 24 (2), pp.12733. http://dx.doi.org/10.11606/issn.2238-6149.v24i2p127-133

Rebellato C., Emmel M.L.G., Cordeiro J.J.R., Oishi J. (2015). Factors associated with occupational roles in older Brazilians: a cross-sectional pilot study. Cad. Ter. Ocup. UFSCar, São Carlos, 23(3), pp. 499513. http://dx.doi.org/10.4322\%2F0104-4931.ctoAO0638

Salles M.M., Matsukura T.S. (2013). Estudo de revisão sistemática sobre o uso do conceito de cotidiano no campo da terapia ocupacional no Brasil. Cadernos de Terapia Ocupacional da UFSCar, 21 (2), pp 265-273. http://dx.doi.org/10.4322\%2Fcto.2013.028

Santi A., Mariotti M.C., Cordeiro J.J.R. (2012). Lista de Identificação de Papéis Ocupacionais em um centro de tratamento de Hemodiálise: contribuições para a intervenção de Terapia Ocupacional - estudo piloto. Revista de Terapia Ocupacional, Universidade de São Paulo, 23 (3), pp. 289-96. http://dx.doi.org/10.11606/issn.22386149.v23i3p289-296

Santos W.R. (2008). Pessoas com deficiência: nossa maior minoria. Physis [online]; 18(3), pp.501-519. http://dx.doi.org/10.1590/S010373312008000300008

Secretaria Nacional de Promoção dos Direitos da Pessoa com Deficiência (SNPD). Cartilha do Censo 2010 - Pessoas com Deficiência. Brasília: SDH-PR/SNPD (2012). Recuperado en: http://www.pessoacomdeficiencia.gov.br/app/sites/default/files/publicacoes/cartiIha-censo2010-pessoas-com-deficienciareduzido.pdf

Souza F.D.A., Cruz D.M.C., Ferrigno I.S.V., Tsukimoto G.R., Figliolia C.S (2013). Correlação entre papéis ocupacionais e independência de usuários com lesão medular em processo de reabilitação. O Mundo da Saúde (CUSC. Impresso), 37, p. 166-175.

Souza F.R.S., Pimentel A.M. (2012). Pessoas com deficiência: entre necessidade e atenção à saúde. Cadernos de Terapia Ocupacional UFSCar, 20 (2), pp. 229-237. http://dx.doi.org/10.4322/ cto.2012.024

Tanaka E.D.O., Manzini E.J. (2005). O que o s empregadores pensam sobre o trabalho da pessoa com deficiência? Revista Brasilera de Educacão Especial, 11 (2), pp 22. http://dx.doi.org/10.1590/S141365382005000200008

United Nations (1980). IYDP Plan of action - Resolution 34/158, June 13, 1979. Nova York: Division for Economic and Social Information, 29 julho 1980. 